\title{
DWT-DCT-SVD Based Semi Blind Image Watermarking Using Middle Frequency Band
}

\author{
Arpita Srivastava ${ }^{1}$, Praful Saxena ${ }^{2}$ \\ ${ }^{I}$ (IFTM University, Moradabad,India) \\ ${ }^{2}$ (KIMT , Moradabad,India)
}

\begin{abstract}
In this paper a semi-blind algorithm is been developed using DWT-DCT and SVD technique which is robust against several attacks like cropping, noise, rotation, filtering, translation, etc. Trigonometric function is used to closely relate the singular values of the original image and the watermarked image. In this algorithm, firstly DWT is applied on the host image which results in four frequency bands $L L, L H, H L$ and $H H$. As it is found that middle frequency band is less prone to attacks so the singular values of the DCT Transformed coefficients of the LH band of the image is been modified using the singular values of the DCT transformed coefficients of the watermark and the scaling factor with the help of inverse-trigonometric function. And then this modified singular values are used to reconstruct the watermarked Host Image. Now to validate the content authentication, the extraction technique is applied on the watermarked image. It consists of applying DWT on the watermarked image to get all the four frequency bands and then by using the singular values of the DCT coefficients of the middle frequency band and the scaling factor using trigonometric function, the singular values of the watermark are extracted to reconstruct the watermark using inverse SVD.
\end{abstract}

Keywords- DCT, DWT, Robust, Semi Blind Watermarking

\section{Introduction}

The evolution of internet, along with the advancement of digital multimedia tools have create a major impact in making the storage and distribution of multimedia content a straightforward tasks. Thus security of multimedia contents becomes a vital issue and there is a need in protecting the digital content against counterfeiting, piracy and malicious manipulations[2]. Digital watermarking is an evolving field that requires continuous effort to find for the best possible method in protecting multimedia content. In this paper, we describe overview of digital image watermarking technique and proposed an algorithm for copy write protection of digital images in DCT domain. Digital watermarking can be defined as the process of embedding a certain piece of information, technically known as watermark into multimedia content including text documents, images, audio or video streams, such that the watermark can detected or extracted later to make an assertion about the data. We have also implemented this algorithm using MATLAB 7.x . Watermark robustness is one of the major characteristics that influence the performance and applications of digital image watermarks[1]. The major advantage of transform domain methods is their superior robustness to common image distortions. In this paper, we are interested in invisible watermarks because they have a wider range of applications compared to visible watermarks.

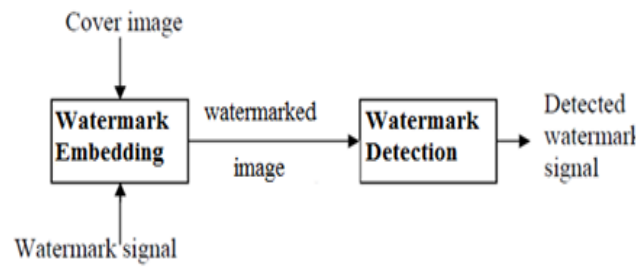

Fig1: Generic Watermarking Scheme

The aims of this paper are three fold:

II. Aims

i. To investigate the strength and limitations of current watermarking schemes .

ii. To design and develop new schemes to overcome the limitations.

iii. To evaluate the new schemes using application scenarios of copyright protection, tamper detection and authentication. 
Aimed at three goals mentioned above, we will find answers to these research questions:

i. What are the major challenges in robust watermarking?

ii. How can we reduce the computational cost of a robust watermarking method that is based on semiblind scheme using trigonometric function?

iii. What are the new capabilities of robust watermarking?

\section{Prior Art}

Three essential factors used to determine quality of watermarking scheme:

\subsection{Discrete Cosine Transform ( DCT)}

The Discrete Cosine Transform (DCT) is a technique that converts a spatial domain waveform into its constituent frequency components as represented by a set of coefficients. DCT is one of the most popular linear transforms and a compression tool of digital signal processing[4]. It is a lossless procedure and has been widely used because of its good capacity of energy compression and de-correlation. These transforms are the members of real-valued discrete sinusoidal unitary transforms.

For $\mathrm{u}, \mathrm{v}=0,1,2 \ldots \ldots \mathrm{N}-1$

$$
C(u, v)=\alpha(u) \alpha(v) \sum_{x=0}^{N-1} \sum_{y=0}^{N-1} f(x, y) \cos \left[\frac{(2 x+1) u \pi}{2 N}\right] \cos \left[\frac{(2 y+1) v \pi}{2 N}\right]
$$

WHERE

$$
\alpha(u)=\left\{\frac{1}{\sqrt{2}} \text { for } u=0,1 \text { for } u=1,2, \ldots . . N-1 . \alpha(v)=\left\{\frac{1}{\sqrt{2}} \text { for } v=0,1 \text { for }=1,2, \ldots . . N-1 .\right.\right.
$$

\subsection{Discrete wavelet Transform ( DWT)}

This watermarking method is focusing on its embedding strength would provide useful insight in how to improve its performance. The performances measured include robustness, imperceptibility and computational cost.DWT decomposes an image into a low-pass sub band and three high- pass sub bands. In this study, this method embeds a watermark in high-pass (or higher frequency) band of the DWT domain[5]. This is due to the good imperceptibility provided by high-pass band. To reconstruct an image, an inverse DWT is used after modification has been made by using singular values of SVD of watermark.

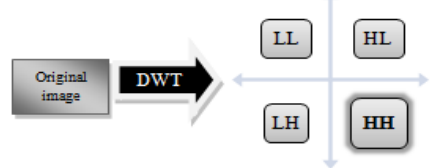

Fig 3: DWT decomposition

\subsection{Singular Value Decomposition( SVD)}

It is a linear algebra transform technique used in watermarking. It is composed of three vectors in which one vector consists of diagonal matrix and two vectors consist of orthogonal matrices. This diagonal matrix is responsible for an image luminance and orthogonal matrices are responsible for the geometry of an image. In this algorithm, the authors found the singular values of cover image and then modified them by adding a watermark[5]. SVD transform is again applied on the resultant matrix for finding the modified singular values. These singular values are combined with the known orthogonal components to get the watermarked image. For watermark extraction, inverse process is used.

\section{Proposed Method}

For the implementation of the proposed algorithm it is assumed that the dimension of the cover image I is $\mathrm{N} \mathrm{x} \mathrm{N}$. First we will embed the watermark in a cover image or host image by using watermark embedding algorithm and then the watermark will be extracted using extraction algorithm. The robustness of the algorithm will me measured by applying attacks on watermarked image and then with the help of PSNR values the robustness of the extracted logo will be evaluated.

\subsection{Watermark Embedding Algorithm}

Step1. Using DWT, decompose the original image I into 4 sub-bands: LL, HL, LH, and HH.

Step 2. Apply DCT on LH sub-band to obtain the coefficient matrix 'A'.

Step 3. Apply SVD on coefficient matrix 'A':

$\mathrm{A}=\mathrm{U}^{\mathrm{h}} \mathrm{S}^{\mathrm{h}} \mathrm{V}^{\mathrm{h}}$

Step 4. Apply DCT on the watermark to obtain the coefficient matrix ' $W$ '.

Step 5. Apply SVD to coefficient matrix ' $W$ ': 
$\mathrm{W}=\mathrm{U}^{\mathrm{w}} \mathrm{S}^{\mathrm{w}} \mathrm{V}^{\mathrm{w}}$

Step 6. Modify $\mathrm{S}^{\mathrm{h}}: \mathrm{S}^{\mathrm{h}^{*}}=\operatorname{tand}^{-1}\left(\alpha^{*} \mathrm{~S}^{\mathrm{w}}\right)$ where $\alpha$ is scaling factor.

Step 7. Obtain the modified high frequency matrix component $\mathrm{I}^{*}{ }^{*}$

$\mathrm{I}^{* \mathrm{~h}}=\left[\mathrm{U}^{\mathrm{h}} \cdot \mathrm{S}^{\mathrm{h}^{*}} \cdot \mathrm{V}^{\mathrm{h}}\right]$

Step 8. Apply IDCT on matrix I* ${ }^{* h}$ to obtain the modified middle frequency band $\mathrm{LH}^{*}$.

$\mathrm{LH}^{*}=\operatorname{IDCT}\left(\mathrm{I}^{* \mathrm{~h}}\right)$

Step 9. Using LL, HL, LH, and $\mathrm{HH}^{*}$, apply IDWT to obtain the watermarked image I*.

The result of embedding algorithm is shown in figure below:

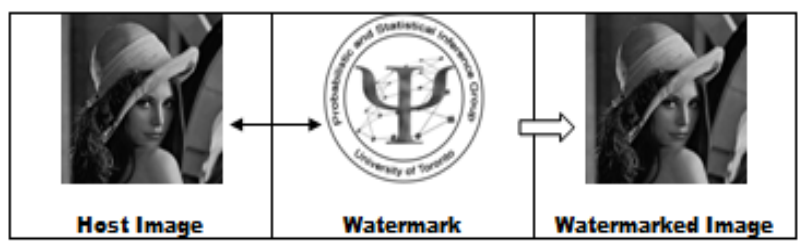

Fig 4: Embedding Process

\subsection{Watermark Extraction Algorithm}

Step1. Using DWT, decompose the watermarked image I* into 4 sub-bands: LL, HL, LH and HH.

Step 2. Apply DCT on LH band to obtain the coefficient matrix ' $\mathrm{C}$ '.

Step 3. Apply SVD to matrix 'C':

$\mathrm{C}=\left[\mathrm{U}^{\mathrm{k}} \mathrm{S}^{\mathrm{k}} \mathrm{V}^{\mathrm{k}}\right]$

Step 4. Apply DCT on watermark W to obtain a coefficient matrix A2.

Step 5. Apply SVD to matrix A2:

$\mathrm{W}^{*}=\left[\mathrm{U}^{\mathrm{w}} \mathrm{S}^{\mathrm{w}} \mathrm{V}^{\mathrm{w}}\right]$

Step 6. Extract the singular values of watermarking:

$\mathrm{S}^{\mathrm{w} *}=\left[\operatorname{tand}\left(\mathrm{S}^{\mathrm{k}}\right)\right] / \alpha$

Where $S^{k}$ are the singulars of watermarked image after applying DCT. [ $\left.\alpha=0.00015\right]$

Step 7. Recover the watermark: $\mathrm{w}^{*}=\left(\mathrm{U}^{\mathrm{w}}\right) *\left(\mathrm{~S}^{\mathrm{w}^{*}}\right) *\left(\mathrm{~V}^{\mathrm{w}}\right)$

Step 8. Apply IDCT on w* to obtain an extracted watermark WM.

Results of various attacks applied on the watermarked image and watermark extracted with its PSNR values is given in the following table:

Table 4: Results of Watermark Extraction after applying various attacks

\begin{tabular}{|c|c|c|c|c|}
\hline \multirow[t]{2}{*}{$\begin{array}{l}\text { ATTACKS } \\
\text { (Parameters) }\end{array}$} & \multirow[t]{2}{*}{$\begin{array}{l}\text { Attacked Watermarked } \\
\text { Image }\end{array}$} & \multirow[t]{2}{*}{ Watermark } & \multicolumn{2}{|c|}{ PROPOSED METHOD } \\
\hline & & & PSNR & Correlation \\
\hline \multicolumn{5}{|l|}{ Rotate $\left(75^{\circ}\right)$} \\
\hline & & & $+53.56 \mathrm{~dB}$ & 0.5308 \\
\hline \multicolumn{5}{|l|}{ Medfilt2 } \\
\hline & & & $+53.74 \mathrm{~dB}$ & 0.6019 \\
\hline Histeq & & & & \\
\hline & & & $+65.52 \mathrm{~dB}$ & 0.9123 \\
\hline
\end{tabular}




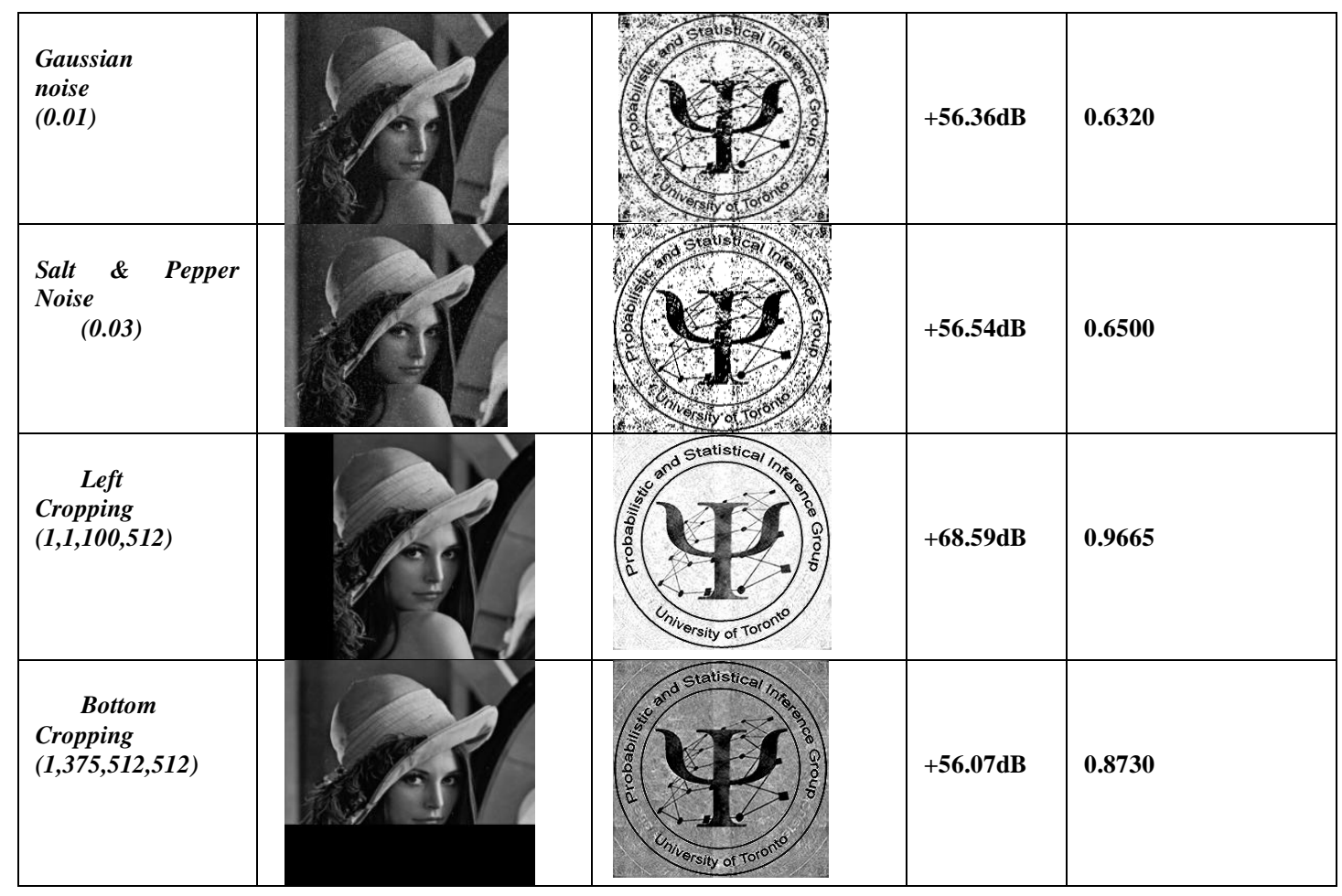

\section{Conclusion}

In this paper, we proposed a semi-blind reference image watermarking by using the technique DWTDCT-SVD. The proposed method has a good performance in the case of robustness and imperceptibility. It is more robust to some common image processing including Gaussian noise, Left cropping, Bottom cropping, Salt \& Pepper noise, Histogram equalization, Medium filter, Rotation, etc. PSNR values are computed to find out the imperceptibility performance between watermarked image and its cover image. The maximum PSNR values is of Left Cropping i.e. $+68.59 \mathrm{~dB}$ and the minimum PSNR values is of Rotation i.e. $+53.56 \mathrm{~dB}$.Correlation values are also computed here for finding the relation between them. The maximum correlation exists for left cropping attack whose value is 0.9665 and the minimum correlation exists for rotation attack whose value is 0.5308 . We compared the results of our proposed method with the existing method of Nidhi H. Divecha and N.N.Jani [3]. The PSNR values and correlation factor of the proposed method are much more robust than the existing one. In the existing method, an original image is of $256 * 256$ pixel values while in the proposed method, we took an original image of $512 * 512$ pixel values.

\section{Acknowledgment}

We take this opportunity to express our sincere thanks and deep gratitude to the authors who extended their whole-hearted co-operation and have helped us in completing this research work successfully.

\section{References}

[1] Emir Ganic and Ahmet M. Eskicioglu,"Robust DWT-SVD Domain Image Watermarking: Embedding Data in all frequencies”,MM\&SEC'04,September 20-21,2004,Magdeburg,Germany,unpublished

[2] Ms. Kapre Bhagyashri S and Mrs. Joshi M.Y.,"Robust Image Watermarking based on Singular Value Decomposition abd Discrete Wavelet Transform",978-1-4-4244-5540-9/10/\$26.00@2010 IEEE,pp.337-341, unpublished.

[3] Nidhi H. Divecha and N.N.Jani,"Image Watermarkin Algorithm using DCT-DWT-SVD”,NCIPET-2012,Proceedings published by International Journal Of Computer Applications® (IJCA),pp.13-16 .

[4] Satyanaryana Murty,M.Uday Bhaskar,P.Rajesh Kumar,“A Semi-Blind Reference Watermarking Scheme Usind DWT-DCT-SVD For Copyright Protection”,International Journal Of Computer Science and Information Technology(IJCSIT), Vol 4,No 2,April 2012,pp. 6982.

[5] Chaw-Seng Woo_ Thesis, "Digital Image Watermarking Methods For Copyright Protection and Authentication",Faculty Of Information Technology,Queensland University Of Technology,March 2007.

[6] Pooya Monshizadeh Naini,"In-Tech Digital_Watermarking_Using Matlab”,University of Tehran,Iran(www.intechopen.com)

[7] M.Thaler, H.Hochreutener,"Image Processing Basics Using Matlab”,February 2008, (C ZHAW. 